\title{
Информационное воздействие на дичность в сети Интернет как один из предикторов частоты рецидивов расстройств пищевого поведения
}

\author{
Юлия В. Свиткевич \\ Южный федеральный университет, г. Ростов-на-Дону, Российская Федерация \\ E-mail: julia.svitkevich@mail.ru \\ ORCID ID: https://orcid.org/0000-0002-7547-5604
}

\section{Аннотация}

В статье рассматривается информационное воздействие на ^ичность в сети Интернет как один из преАикторов частоты рециАивов расстройств пищевого поведения. Во Ввелении автором раскрывается актуальность темы, которая определяется распространенностью расстройств пищевого поведения и растущей популярностью информации в Интернете о питании, похудении, а также романтизацией расстройств пищевого поведения. Также автором определяются понятия ремиссии и рециАива расстройств пищевого повеАения, объясняется патогенез заболевания. Новизна исследования заключается в рассмотрении информационного возАействия на ^ичность в сети Интернет в качестве предиктора частоты рециАивов расстройств пищевого поведения. В разАеле ॥ОбсужАение результатов І автор акцентирует внимание на тех результатах исслеАования, которые полтвержАают влияние инорормации на личность, страдающую от расстройства пищевого поведения. Автор отмечает, что информация из неавторитетных источников положительно коррелирует с наличием симптомов расстройства пищевого поведения. НеожиАанным выводом становится то, что вне зависимости от категории используемых источников инорормации, респонАенты имеют схожую выраженность симптомов. В Заключении Аелаются выводы О том, что: 1) существует выраженная связь межАу инорормационным воздействием на ^ичность и выраженностью симптомов расстройств пищевого повеАения; 2) иинфрормационное воздействие на личность может быть преАставлено в виде пропаганды излишней худобы в качестве илеала образа тела, романтизацией расстройств пищевого поведения, а также пропаганАой некорректных способов похудения; 3) среди тех, кто участвует в пропагандирующих расстройства пищевого поведения сообществах, сильнее выражены симптомы расстройств пищевого повеАения; 4) выбор источников инорормации зависит от возраста респонАента; 5) авторитетные источники инфрормации (рекоменАации ВОЗ, РАМН, библиотечные системы ScienceDirect, PubMed) практически не используются респондентами; 
6) деструктивное инорормационное воздействие на пищевое поведение может осуществляться ^атентно, вне зависимости от направленного изучения ^ичностью правливой информации о похудении.

\title{
КАючевые слова
}

расстройства пищевого поведения, похудение, инфоормация, инорормационное воздействие, интернет, социальные сети, сообщества, источники информации

\section{Для цитирования}

Свиткевич Ю.В. Информационное воздействие на дичность в сети Интернет как один из предикторов частоты рецидивов расстройств пищевого поведения // Инновационная наука: психология, педагогика, дефектология. 2021. Т. 4, № 2. C. 32-47. doi: https://doi.org/10.23947/2658-7165-2021-4-2-32-47

\section{Information impact on the personality on the Internet as one of the predictors of the frequency of relapses of eating disorders}

\author{
Yulia V. Svitkevich \\ Southern Federal University, Rostov-on-Don, Russian Federation \\ E-mail: julia.svitkevich@mail.ru \\ ORCID ID: https://orcid.org/0000-0002-7547-5604
}

\begin{abstract}
The article considers the information impact on the individual on the Internet as one of the predictors of the frequency of relapses of eating disorders. In the introduction, the author reveals the relevance of the topic, which is determined by the prevalence of eating disorders and the growing popularity of information on the Internet about nutrition, weight loss, as well as the romanticization of eating disorders. The author also defines the concepts of remission and relapse of eating disorders, explains the pathogenesis of the disease. The novelty of the study is to consider the information impact on the individual on the Internet as a predictor of the frequency of relapses of eating disorders. In the section «Discussion of results», the author focuses on the results of the study confirming the impact of information on a person suffering from an eating disorder. The author notes that information from non-authoritative sources positively correlates with the presence of symptoms of an eating disorder. An unexpected conclusion is that, regardless of the category of information sources used, respondents have a similar severity of symptoms. In conclusion, it is concluded that: 1) there is a pronounced relationship between the informational impact on the individual and the severity of symptoms of eating disorders; 2 ) the informational impact on the individual
\end{abstract}


ПСИХОЯОГИЯ

can be represented in the form of propaganda of excessive thinness as an ideal of body image, romanticization of eating disorders, as well as propaganda of incorrect ways to lose weight; 3 ) among those who participate in communities promoting eating disorders, the symptoms of eating disorders are more pronounced; 4) the choice of information sources depends on the age of the respondent; 5) authoritative sources of information (recommendations of the WHO, RAMS, library systems ScienceDirect, PubMed) are practically not used by respondents; 6) destructive information impact on eating behavior can be carried out latently, regardless of the person's directed study of truthful information about weight loss.

\section{Keywords}

eating disorders, weight loss, information, information impact, internet, social networks, communities, information sources

\section{For citation}

Svitkevich, Yu. V. (2021). Information impact on the personality on the Internet as one of the predictors of the frequency of relapses of eating disorders. Innovative Science: psychology, pedagogy, defectology, 4(2), 32-47. doi: https://doi. org/10.23947/2658-7165-2021-4-2-32-47

\section{Введение}

Современные тенденции общества: компьютеризация и глобализация. Значительно возросла значимость Интернета. Его теперь используют повсеместно. Одна из самых используемых областей - социальные сети и медиа, общение. Несомненно, передача сообщений по сети полезна, но последствия продолжительного использования Интернета носят всё-таки амбивалентный характер.

Вместе с этим образ жизни современного человека тоже претерпел изменения: существенно сократились энергозатраты большинства людей, а пищевая индустрия предлагает все новые продукты с уникальными вкусовыми качествами и человек постоянно испытывает новые соблазны. Результатом всего этого стало распространение ожирения. Данные ВОЗ также показывают, что во всем мире насчитывается более 1 миллиарда взрослых людей с избыточным весом и 300 миллионов людей, страдающих ожирением (Sattar, et. al., 2008). Обратная сторона ожирения - это голодание, анорексия, избегание пищи, частично расстройства пищевого поведения.

Расстройства пищевого поведения (РПП) - это клинические явления, которые вызывают все больший интерес среди специалистов в связи с их распространением (Moreno, et. al., 2012). С 70-х годов до настоящего времени было проведено много исследований РПП, и в настоящее время они представляют собой категорию в рамках международных классификаций психических заболеваний. 
Закономерно увеличилась и распространенность расстройств пищевого поведения. Так, заболеваемость нервной анорексией увеличилась уже в 50 раз с 1930-х годов (Hoek, 2006). Некоторые исследования свидетельствуют о продолжающемся росте заболеваемости среди молодого населения (Zipfel, Giel, Bulik, Hay \& Schmidt, 2015).

Сейчас при диагностировании РПП используются: диагностическое и статистическое руководство по психическим расстройствам 5-го издания (DMS-V), международная классификация болезней 10-го пересмотра (МКБ-10). В свою очередь, классификация DMS-V, принятая в 2013 году, существенно расширила описание и дифференциацию расстройств пищевого поведения. B DMS-IV было описано только 3 диагноза РПП: нервная анорексия (НА), нервная булимия (НБ) и неуточненное расстройство пищевого поведения (НРПП).

Патогенез расстройства пищевого поведения сопровождается сменой эпизодов ремиссии - отсутствия всех симптомов или надичия 1-2 остаточных симптомов в течение 8 недель (Keel, Dorer, Franko, Jackson \& Herzog, 2005) и рецидива по крайней мере один эпизод переедания-очищения в неделю иди пищевых ограничений, показатель ИМТ < 17,5 3 месяца подряд (Carter, et. al., 2012). Само развитие расстройств пищевого поведения женщинами в одном из исследований объясняется отсутствием контроля, чувством отстраненности от семьи и сверстников. Восстановление же характеризуется ими тем, что женщины снова занялись жизнью, развили навыки, необходимые для разрешения конфликтов, и вновь обрели чувство собственной полноценности (Patching \& Lawler, 2009).

Редко полное выздоровление от расстройств пищевого поведения. Так, в одном из исследований оценка клинического и психологического выздоровления у пациентов с расстройством пищевого поведения через 5 лет после начала лечения в специализированном амбулаторном отделении показало, что доля полного выздоровления, т. е. в дополнение к субъективному отчету о том, что пациент полностью выздоровел и не имеет никаких физических, поведенческих или психодогических симптомов расстройства пищевого поведения, составила всего 40,8 \% (Isomaa \& Isomaa, 2014).

Актуальность проблемы связана с распространенностью расстройств пищевого поведения и, что самое важное, самой высокой смертностью среди пациентов с психическими заболеваниями (Arcelus, Mitchell, Wales \& Nielsen, 2011), а также распространением текстовых источников в сети Интернет, посвященной теме питания, похудения и расстройствам пищевого поведения. Становится все больше заманчивых, интригующих или не соответствующих действительности заголовков статей. Таких как, например: «Секрет похудения? Ешьте больше!», «Переход к тренажерный зал может сделать вас толстым», «Употребление большего количества жира при сокращении углеводов и отказе от сахара может помочь вам похудеть и стать счастливее, говорит ведущий кардиолог». Такие заголовки 
ПСИХОЛОГИЯ

предполагают, что состав диеты, независимо от энергетического баланса, определяет потерю или увеличение веса - это не соответствует имеющимся научным данным о том, что только состояние энергетического баланса вдияет на потерю или набор массы (Gonzalez, 2018).

Воздействие искаженной информации вдиять на подсознание личности и ее поведение. В свою очередь, распространителями дезинформации, по мнению многих авторов, являются социальные сети: «Одним из основных распространителей дезинформации, обсуждаемых в литературе, были социальные медиа, которые облегчают разрастание нерегулируемой и непроверенной информации, которая не соответствует первоначальным источникам» (De Paor \& Heravi, 2020 , с. 7). Таким образом, распространенность РПП отчасти связана с развитием глобальной сети. Так, например, изображение тела, просматриваемого в Интернете, может генерировать чувство удовлетворенности или неудовлетворенности собственной фигурой (Fichter, et. al., 2012). Члены Интернет-сообществ вступают в конкуренцию, чтобы потерять как можно больше веса. Члены этих сообществ используют худые образы тела в качестве вдохновения (Mento, et. al., 2021). В одном из исследований была показана взаимосвязь между исподьзованием Интернета и симптомами расстройств пищевого поведения, в частности: неудовлетворенностью телом, ограничительным питанием и стремлением к худобе (Ioannidis, et. al., 2020).

Результатом становится то, что многие пользователи социальных сетей не понимают, соответствует ли информация действительности и чему на самом деле стоит доверять. Действительно, информацию можно проверять с помощью специальных моделей поведения и приемов информационной грамотности. Однако большинство пользователей Интернета не имеют навыков цифровой грамотности и поэтому не могут принимать обоснованные решения (Torres, Gerhart \& Negahban, 2018). Это еще раз подтверждает актуальность исследования проблемы воздействия информации в интернете на дичность.

Целью данного исследования становится изучение способов информационного воздействия на личность в сети Интернет, а также связь этого воздействия с выраженностью симптомов и вероятностью рецидива расстройства пищевого поведения.

\section{Методы}

В исследовании приняли участие 64 человека: 61 женщина и 3 мужчины. Мы исподьзовали метод анкетирования в онлайн-среде с помощью платформы «Google Forms». Нами был составлен опросник, состоящий из 25 вопросов.

Нами была выдвинута гипотеза о том, что Аюди, состоящие в сообществах, пропагандирующих способные навредить здоровью способы похудения, будут иметь симптомы расстройств пищевого поведения и не будут набдюдаться 
у специалистов психологов и диетологов. Также мы предположили, что участники тематических интернет-сообществ о похудении будут доверять дезинформации, предоставляемой авторами блогов и распространять ее входе обсуждений на форумах, в чатах и в дичных сообщениях.

Таким образом, нас интересовало следующее:

1) Предпочитаемые респондентами источники информации о похудении и коррекции фигуры.

2) Вовлеченность респондентов в тематические сообщества в социальных сетях, посвященные расстройствам пищевого поведения (например, «Типичная анорексичка», «Типичная булимичка» и т. д.).

3) Зависимость выраженности симптомов расстройств пищевого поведения от используемых источников у опрошенных респондентов.

Выборка была сформирована случайным образом. Мы дали объявление об исследовании и попросили всех желающих принять в нем участие. Объявление размещалось в сообществах в социальных сетях: «Типичная анорексичка», «Голод», «Дечебное голодание», «Типичная булимичка», «90-60-90», а также на форуме проекта «Мой здоровый рацион».

\section{Результаты}

Для усреднения полученных данных мы искдючиди ответы респондентов мужского пола, поэтому для анализа остался 61 ответ. Мы также разделиди респондентов на 3 возрастные категории:

1. Подростки $(12-17$ лет) $=23$ человека.

2. Молодые $(18-30$ лет $)=18$ человек.

3. Взрослые $(30+$ лет) $=20$ человек.

Результаты исследования показали, что информацию о коррекции фигуры $78.7 \%$ ( $\mathrm{n}=48)$ опрошенных заимствуют из постов, блогов в социальных сетях. Примерно также востребованы оказались статьи в Интернете - $73.8 \%(n=45)$. Меньше всего респонденты были заинтересованы в изучении авторитетных источников. Только $3.3 \%(n=2)$ опрошенных ответили, что используют в качестве источников информации рекомендации организации питания РАMН и столько же ответили, что читают статьи в библиотечных системах ScienceDirect, SCOPUS и т. д. Только $1.6 \%(\mathrm{n}=1)$ используют в качестве источника рекомендации ВОЗ. $8.2 \%(\mathrm{n}=5)$ телевидение, $3.3 \%(\mathrm{n}=2)$ респондентов считают авторитетными источниками газеты и журналы, а $32.8 \%$ (n=20) - книги.

Мы изучади связь между возрастом респондентов и источниками информации, используемой для похудения. В Таблице 1 мы проанализировали используемые респондентами источники информации и возраст отвечавших. Оказалось, что подростки больше всего использовали посты и блоги в социальных сетях $-95.65 \%(n=22)$ и Интернет-ресурсы, сайты - $73.91 \%$ (n = 17), а также 
ПСИХОЛОГИЯ

использовали рекомендации знакомых из Интернет-сообществ - 43.48 \% (n = 10). Примечательно, что среди 23 опрошенных подростков никто не исподьзовал авторитетные источники информации.

\begin{tabular}{|c|c|c|c|}
\hline \multicolumn{4}{|c|}{$\begin{array}{l}\text { Таблица } 1 \\
\text { Используемые респондентами источники инорормации Аля похудения }\end{array}$} \\
\hline Источник инорормации & $\frac{\text { ПоАростки }}{(n=23)}$ & $\frac{\text { Mолодые }}{\underline{(n=18)}}$ & $\begin{array}{c}\text { Взрослые } \\
(n=20)\end{array}$ \\
\hline $\begin{array}{l}\text { Посты и блоги в социальных } \\
\text { сетях }\end{array}$ & $95.65 \% / 22$ & $88.89 \% / 16$ & $45 \% / 9$ \\
\hline Интернет-ресурсы & $73.91 \% / 17$ & $88.89 \% / 16$ & $30 \% / 6$ \\
\hline $\begin{array}{l}\text { Рекомендации знакомых } \\
\text { из Интернет-сообществ }\end{array}$ & $43.48 \% / 10$ & $27.78 \% / 5$ & $15 \% / 3$ \\
\hline Книги & $21.74 \% / 5$ & $38.89 \% / 7$ & $35 \% / 7$ \\
\hline $\begin{array}{l}\text { Аругое (журналы, } \\
\text { телевиление, газеты) }\end{array}$ & $8.7 \% / 2$ & $5.56 \% / 1$ & $15 \% / 3$ \\
\hline $\begin{array}{l}\text { Библиотечные системы } \\
\text { и базы } А \text { анных }\end{array}$ & 0 & $5.56 \% / 1$ & $5 \% / 1$ \\
\hline РекоменАации ВОЗ и РАМН & 0 & $5.56 \% / 1$ & $5 \% / 1$ \\
\hline
\end{tabular}

Результаты исследования, приведенные в Таблице 2, показали, что в нашей выборке наибольшее количество участников сообществ «Типичная анорексичка», «90-60-90», «Типичная булимичка»- подростки, $91.3 \%$ ( $\mathrm{n}=21)$. В таких сообществах состоят $77.78 \%(\mathrm{n}=14)$ от кодичества молодых респондентов и только $25 \%(\mathrm{n}=5)$ из числа взрослых отвечавших. 


\begin{tabular}{|lccc|}
\hline Таблица 2 & & \\
Участие разных возрастных групп респондентов в сообществах о похудении
\end{tabular}

Мы задали вопросы, касающиеся отношения респондентов к информации в Интернете. Полученные результаты отображены в Таблице 3. Позитивным наблюдением было то, что 73,8 \% (n = 45) опрошенных проверяют информацию, полученную в блоге или на сайте. Чуть более половины опрошенных респондентов $-54,1$ \% ( $=33)$ считают, что информация о похудении в Интернете достаточно запутанна.

\begin{tabular}{|c|c|c|c|}
\hline \multicolumn{4}{|c|}{$\begin{array}{l}\text { Таблица } 3 \\
\text { Отношение респондентов к инорормации в Интернете }\end{array}$} \\
\hline Позиция & ПоАростки $(n=23)$ & $\frac{\text { Mолодые }}{\underline{(n=18)}}$ & Взрослые $(n=20)$ \\
\hline $\begin{array}{l}\text { Инорормация } \\
\text { понятна }\end{array}$ & $39.13 \% / 9$ & $66.67 \% / 12$ & $35 \% / 7$ \\
\hline $\begin{array}{l}\text { Инорормация } \\
\text { запутанна }\end{array}$ & $60.87 \%$ / 14 & $33.33 \% / 6$ & $65 \% / 13$ \\
\hline $\begin{array}{l}\text { Проверяют } \\
\text { инорормацию }\end{array}$ & $78.26 \% / 18$ & $66.67 \% / 12$ & $75 \% / 15$ \\
\hline
\end{tabular}


ПСИХОЛОГИЯ

Наше исследование также было направлено на изучение симптомов расстройств пищевого поведения в контексте вдияния на индивида информации в социальных сетях и Интернет-пространстве. Мы предположили, что респонденты, состоящие в публичных сообществах наподобие «Типичная анорексичка», будут иметь больше симптомов расстройства пищевого поведения. Поэтому мы проанализировади общее количество респондентов с симптомами и выделиди из общего кодичества тех, кто состоит в соответствующих сообществах.

Таким образом, из общего количества респондентов, намеренно вызывающих рвоту после переедания чаще, чем раз в неделю $82.35 \%(n=14)$ состояли в указанных тематических сообществах. Также от общего кодичества сознательно потребляли меньше калорий, чем необходимо для безопасного похудения, $83.33 \%$ (n = 15) респондентов, состоящих в указанных группах. Используют средства «для похудения» $50.82 \%(\mathrm{n}=31)$ всех опрошенных и $77.42 \%(\mathrm{n}=24)$ из отвечавших состоят в сообществах. Однако, тодько $62.71 \%$ (n = 37) от общего количества опрошенных, неудовлетворенных своим телом, участвуют в тематических сообществах.

Корреляция между выраженностью симптомов расстройств пищевого поведения и участие в тематических сообществах отображена в Таблице 4.

\begin{tabular}{|c|c|c|}
\hline \multicolumn{3}{|c|}{$\begin{array}{l}\text { Таблица } 4 \\
\text { Симптомы РПП среди общего кол-ва респондентов и участников сообществ } \\
\text { «Типичная анорексичка», «Типичная булимичка», «90-60-90» }\end{array}$} \\
\hline Симптомы & Bсего $(n=61)$ & $\begin{array}{l}\text { В том числе участники } \\
\text { групп из общего кол-ва }\end{array}$ \\
\hline $\begin{array}{l}\text { Самоиндуцированная } \\
\text { рвота чаще, чем раз } \\
\text { в нелелю }\end{array}$ & $27.87 \% / 17$ & $82.35 \%$ / 14 из 17 \\
\hline Боязнь потолстеть & $86.89 \% / 53$ & $66.04 \%$ / 35 из 53 \\
\hline $\begin{array}{l}\text { Средства Аля } \\
\text { похудения } \\
\text { (мочегонные, } \\
\text { слабительные) }\end{array}$ & $50.82 \% / 31$ & $77.42 \%$ / 24 из 31 \\
\hline $\begin{array}{l}\text { Сознательное опасное } \\
\text { низкое потребление } \\
\text { калорий (менее 1000) }\end{array}$ & $29.51 \% / 18$ & $83.33 \%$ / 15 из 18 \\
\hline
\end{tabular}




\begin{tabular}{|c|c|c|}
\hline \multicolumn{3}{|c|}{$\begin{array}{l}\text { Таблица } 4 \\
\text { Симптомы РПП среди общего кол-ва респондентов и участников сообществ } \\
\text { «Тпичная анорексичка», «Типичная булимичка», «90-60-90» }\end{array}$} \\
\hline Симптомы & Всего $(n=61)$ & $\begin{array}{l}\text { В том числе участники } \\
\text { групп из общего кол-ва }\end{array}$ \\
\hline $\begin{array}{l}\text { Похудение с помощью } \\
\text { строгих Аиет (моно- } \\
\text { Аиеты, АВС, питьевая } \\
\text { миета, }\end{array}$ & $65.57 \% / 40$ & $75 \%$ / 30 из 40 \\
\hline $\begin{array}{l}\text { НеуАовлетворенность } \\
\text { телом }\end{array}$ & $96.72 \% / 59$ & 62.71 \% / 37 из 59 \\
\hline
\end{tabular}

Нас интересовала связь между видом источников, которые исподьзуют респонденты, и выраженностью симптомов расстройства пищевого поведения. В зависимости от исподьзуемых источников мы также разделиди респондентов на 2 категории:

1. Те, кто указали в качестве используемой информации только статьи на сайтах, а также посты и блоги пользователей в социальных сетях;

2. Те, кто кроме Интернета исподьзует информацию из книг, библиотечных систем, телевидения, газет и журналов.

Нас интересовади некоторые ответы респондентов из этих двух групп. В таблице 5 мы продемонстрировали результаты этих выборок на предмет исподьзования строгих диет, средств для похудения, участия в тематических сообществах и на наличие симптомов расстройства пищевого поведения.

Согласно полученным результатам, средства для похудения (мочегонные и слабительные) использовало $93.94 \%$ (n = 31) опрошенных, которые используют только интернет-ресурсы в качестве источников информации о похудении; и только $46.43 \%$ (n = 13) из тех, кто также исподьзует научные базы данных и книги. Состоят в тематических сообществах типа «Типичная анорексичка», «Типичная булимичка» и т. д. 75.76 \% ( $(\mathrm{n}=25)$ первой группы и 53.57 \% (n=15) второй группы. В целом результаты по другим вопросам не сильно отличаются между группами. Так, произвольно вызывают рвоту чаще, чем раз в неделю $33.33 \%$ (n = 11) респондентов, использующих только информацию из Интернета и $21.43 \%(\mathrm{n}=6)$, исподьзующих, помимо того, научные базы данных и книги. Диеты придерживаются $60.61 \%(\mathrm{n}=20)$ и $60.71 \%(\mathrm{n}=17)$ из первой и второй групп опрошенных соответственно. 
Инновационная наука: психология. педагогика. дефектология 2021 TОМ 4 № 2

ПСИХОЯОГИЯ

\begin{tabular}{|c|c|c|}
\hline \multicolumn{3}{|c|}{$\begin{array}{l}\text { Зависимость ответов респонАентов от того, инорормацию из каких источников } \\
\text { они использУют }\end{array}$} \\
\hline & $\begin{array}{l}\text { Те, кто использует } \\
\text { только Интернет- } \\
\text { ресурсы }(n=33)\end{array}$ & 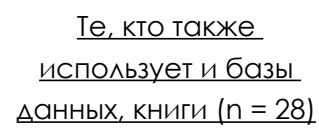 \\
\hline $\begin{array}{l}\text { Похудение с помощью } \\
\text { строгих Аиет (моно-диеты, } \\
\text { АВС, питьевая диета) }\end{array}$ & $69.7 \% / 23$ & $64.29 \% / 18$ \\
\hline $\begin{array}{l}\text { Средства Аля похудения } \\
\text { (мочегонные, } \\
\text { слабительные) }\end{array}$ & $93.94 \%$ / 31 & $46.43 \% / 13$ \\
\hline Считают ^и ка^орийность & $66.67 \% / 22$ & $50 \% / 14$ \\
\hline $\begin{array}{l}\text { Участники сообществ } \\
\text { типа ТА, ТБ, 90-60-90 и т. А. }\end{array}$ & $75.76 \% / 25$ & $53.57 \% / 15$ \\
\hline $\begin{array}{l}\text { Самоиндуцированная } \\
\text { рвота чаще, чем раз } \\
\text { в неделю }\end{array}$ & $33.33 \% / 11$ & $21.43 \% / 6$ \\
\hline $\begin{array}{l}\text { Сознательное опасное } \\
\text { низкое потребление } \\
\text { калорий (менее 1000) }\end{array}$ & $36.36 \% / 12$ & $21.43 \% / 6$ \\
\hline $\begin{array}{l}\text { Не считают инорормацию } \\
\text { в Интернете запутанной }\end{array}$ & $45.45 \% / 15$ & $42.86 \% / 12$ \\
\hline СоблюАают Аиету & $60.61 \% / 20$ & $60.71 \% / 17$ \\
\hline
\end{tabular}

Отдельно необходимо проанадизировать пункт «комментарии». В опросе мы предоставили респондентам возможность высказаться иди как-дибо дополнить данные ими ответы. Нами были полученные следующие комментарии касательно переедания:

- «переедание на фоне нервирования в семье»;

- «переедание обычно в праздники, такие как, например, день рождения»;

- «переедаю, когда быстро ем или на празднике, где много вкусностей». 
У некоторых респондентов переедание вызвано особыми условиями, которые ими осознаются. Это говорит об определенном уровне осознанности.

Самыми интересными комментариями нам показадись следующие:

- «У меня булимия, психолог завел в тупик. Все стороны своего состояния осознаю и стараюсь изменить, т. к. имею высшее медицинское образование, но внутренне не могу побороть желание сбросить вес и эпизоды зажоров». - «После переедания бегу сразу на весы... каждые пять минут подхожу к зеркалу, начинается истерика (вдруг наберу) и далее голод, иногда я вызываю рвоту».

- «Я просто ничего не ем».

Оставившие их респонденты осознают свое поведение и страдают от состояния, в котором находятся. Они указали, что проводят в Интернете в среднем 5-8 ч в сутки, пытались вызывать рвоту после переедания, указали в качестве приоритетной информации посты и блоги в социальных сетях.

\section{Обсуждение результатов}

Результатами исследования подтверждена гипотеза о том, что участники будут иметь симптомы расстройства пищевого поведения, озабоченность весом или неудовлетворенность собственным телом. Таким образом, $96,7 \%$ ( $n=59)$ от общего числа респондентов отметили неудовлетворенность своим телом. 86,9\% (n = 53) респондентов отметиди, что имеют страх потодстеть и $36.07 \%$ (n = 22) периодически этому пытаются препятствовать, вызывая рвоту, а 50,8 \% (n = 31) опрошенных отметили, что до сих пор исподьзуют средства для похудения (мочегонные, слабительные). Как и ожидалось, неавторитетные источники оказались наиболее предпочтительны респондентам.

Нами выдвинута гипотеза о связи используемых респондентами источниках информации и выраженности симптомов расстройства пищевого поведения. Предположительно, менее осознанные и здоровые способы похудения будут положительно коррелировать с исподьзованием искдючительно неавторитетных источников: постов в социальных сетях, непрофессиональных статей из Интернета. Наоборот, дюди, которые бы использовали авторитетные источники: научные статьи, рекомендации Всемирной организации здравоохранения (BO3) и институт питания Российской академии медицинских наук (РАМН). Такие источники информации, как телевидение, газеты и журналы относятся, по нашему мнению, к менее авторитетным источникам информации. Эта гипотеза подтвердилась лишь частично: в целом, схожее количество респондентов в группе, использующих только интернет-ресурсы в качестве информации о похудении и отвечавших, которые также использовали информацию из книг, имели симптомы расстройства пищевого поведения. Заметное раздичие в ответах по вопросам между группами было 
ПСИХОЛОГИЯ

тодько в исподьзовании мочегонных и слабительных в качестве средств для похудения; участии в тематических сообществах о похудении; подсчете калорий и самоиндуцировании рвоты.

Мы можем предположить, что, в дюбом случае, даже люди, использующие дополнительно информацию из книг и научных баз данных, ежедневно подвергаются информационному воздействию в Интернете и могут исподьзовать нездоровые способы похудения, несмотря на знание достоверной информации. В одном из исследований отражено то, что даже кратковременное (менее 5 минут), воздействие информации может значительно изменить бессознательное поведение людей (Bastick, 2021). Это подтверждает нашу гипотезу о том, что существует связь между информационным воздействием в Интернете на личность и частотой рецидивов расстройства пищевого поведения. Ряд исследований также подтверждает гипотезу о том, что вдияние информации на расстройства пищевого поведения действительно существует и воздействие определенных типов онлайн-контента. Например, исподьзование сайтов и социальных сетей может иметь накапдивающиеся эффекты с течением времени (Hsieh, Hsiao, Yang, Liu \& Yen, 2018; Hummel \& Smith, 2015; Tiggemann \& Slater, 2017).

Почему же люди, имея официальные рекомендации, чаще используют информацию с сайтов и блогов? Стимулом найти интересующую информацию в Интернете становится не тодько простота, но и доверие к источнику, что подтверждается результатами нашего исследования. Это частично согласовывается с полученными нами данными. Так, примерно половина опрошенных (44.3\%, $\mathrm{n}=27$ ) считают, что информация в Интернете не запутанна. Нашим предположением может стать следующее: это происходит потому, что в Интернете информация предоставляется простым языком, структурированно. Также во многих источниках используются «истории успеха», реалистичные истории дюдей, которые похудели с помощью предлагаемых сайтами методик. Так, например, в одном из исследований многие респонденты сообщили, что рекомендации из официальных источников «сбивают с толку, что требуются простые, ясные, конкретные, реалистичные, а в некоторых случаях и индивидуальные рекомендации» (Boylan, Louie \& Gill, 2012, с. 615).

В целом, дезинформация, которая связана со здоровьем и медициной, довольно распространена и популярна как в социальных сетях, так и в целом в Интернет-пространстве. Это подчеркивается другими исследователями (Chou, Oh \& Klein, 2018). Иногда дезинформация не только широко распространена в интернете и социальных сетях, но и бывает часто даже более популярной, чем точная информация (Wang, McKee, Torbica \& Stuckler, 2019). Другой род воздействия на пищевое поведение, например, представленная в одном из исследований (Syed-Abdul, et. al., 2013) пропаганда анорексии в видеородиках YouTube, также становится очень популярна среди молодых зрителей. 
В свою очередь информацию, вдияющую на пищевое поведение человека, можно разделить на три категории:

1) Пропаганда издишней худобы, искажение образа тела;

2) Романтизация и популяризация расстройств пищевого поведения;

3) Информация, пропагандирующая некорректные способы похудения, дезинформация о похудении.

Таким образом, в ходе исследования нами были получены следующие результаты:

1) Существует связь между информационным воздействием на личность и выраженностью симптомов расстройств пищевого поведения.

2) Информационное воздействие на Аичность может быть представлено в виде пропаганды издишней худобы в качестве идеала образа тела, романтизацией расстройств пищевого поведения, а также пропагандой некорректных способов похудения.

3) Большинство предпочитают получать информацию со страниц сайтов о похудении, из блогов и постов в социальных сетях.

4) Абсолютное большинство опрошенных отметили, что они не удовлетворены своим телом и боятся поправиться.

5) Выбор источника информации о похудении зависит от возраста респондентов.

6) Деструктивное информационное воздействие на пищевое поведение может осуществляться иатентно, вне зависимости от направленного изучения личностью правдивой информации о похудении.

\section{Закдючение}

Результаты исследования показали, что воздействие информации из сети Интернет способно вызвать рецидив расстройства пищевого поведения из-за пропаганды издишней худобы, романтизации расстройств пищевого поведения и неправильно истолкованных методов похудения.

Это исследование подтверждает необходимость прододжения изучения вдияния информации на дичность с расстройствами пищевого поведения и предложение мер, направленных на развитие навыков информационной грамотности и осведомленности о правидьном питании среди подьзователей Интернета и социальных сетей.

Автор заявляет об отсутствии конфликта интересов.

\section{Дитература}

Arcelus, J., Mitchell, A. J., Wales, J., \& Nielsen, S. (2011). Mortality rates in patients with anorexia nervosa and other eating disorders: a meta-analysis of 36 
ПСИХОЛОГИЯ

studies. Archives of general psychiatry, 68(7), 724-731. doi: https://doi.org/10.1001/ archgenpsychiatry.2011.74

Bastick, Z. (2021). Would you notice if fake news changed your behavior? An experiment on the unconscious effects of disinformation. Computers in Human Behavior, 116, 106633. doi: https://doi.org/10.1016/j.chb.2020.106633

Boylan, S., Louie, J.C.Y., Gill, T.P. (2012). Consumer response to healthy eating, physical activity and weight-related recommendations: A systematic review. Obesity Reviews, 13(7), 606-617. doi: https://doi.org/10.1111/j.1467-789X.2012.00989.x

Carter, J. C., Mercer-Lynn, K. B., Norwood, S. J., Bewell-Weiss, C. V., Crosby, R. D., Woodside, D. B., \& Olmsted, M. P. (2012). A prospective study of predictors of relapse in anorexia nervosa: implications for relapse prevention. Psychiatry research, 200(2-3), 518-523. doi: https://doi.org/10.1016/j.psychres.2012.04.037

Chou, W. Y. S., Oh, A., \& Klein, W. M. (2018). Addressing health-related misinformation on social media. Jama, 320(23), 2417-2418. doi: https://doi.org/10.1001/ jama.2018.16865

De Paor, S., \& Heravi, B. (2020). Information literacy and fake news: How the field of librarianship can help combat the epidemic of fake news. The Journal of Academic Librarianship, 46(5), 102218. doi: https://doi.org/10.1016/j.acalib.2020.102218

Fichter, M. M., Quadflieg, N., Nisslmüller, K., Lindner, S., Osen, B., Huber, T., \& Wünsch-Leiteritz, W. (2012). Does internet-based prevention reduce the risk of relapse for anorexia nervosa? Behaviour research and therapy, 50(3), 180-190. doi: https://doi. org/10.1016/j.brat.2011.12.003

Gonzalez, J. T. (2018). Using misleading online media articles to teach critical assessment of scientific findings about weight loss. Advances in physiology education, 42(3), 500-506. doi: https://doi.org/10.1152/advan.00065.2018

Hoek, H. W. (2006). Incidence, prevalence and mortality of anorexia nervosa and other eating disorders. Current opinion in psychiatry, 19(4), 389-394. doi: https://doi. org/10.1097/01.yco.0000228759.95237.78

Hsieh, K. Y., Hsiao, R. C., Yang, Y. H., Liu, T. L., \& Yen, C. F. (2018). Predictive effects of sex, age, depression, and problematic behaviors on the incidence and remission of internet addiction in college students: a prospective study. International journal of environmental research and public health, 15(12), 2861. doi: https://doi. org/10.3390/ijerph15122861

Hummel, A. C., \& Smith, A. R. (2015). Ask and you shall receive: Desire and receipt of feedback via Facebook predicts disordered eating concerns. International Journal of Eating Disorders, 48(4), 436-442. doi: https://doi.org/10.1002/eat.22336

Ioannidis, K., Taylor, C., Holt, L., Brown, K., Lochner, C., Fineberg, N. A., \& Czabanowska, K. (2020). Problematic usage of the internet and eating disorders: a multifaceted, systematic review and meta-analysis. medRxiv. doi: https://doi. org/10.1101/2020.08.20.20177535 
Isomaa, R, \& Isomaa, A. L. (2014) And then what happened? A 5-year follow-up of eating disorder patients. Nordic Journal of Psychiatry, 68(8), 567-572. doi: https:// doi.org/10.3109/08039488.2014.892152

Keel, P. K., Dorer, D. J., Franko, D. L., Jackson, S. C., \& Herzog, D. B. (2005). Postremission predictors of relapse in women with eating disorders. The American journal of psychiatry, 162(12), 2263-2268. doi: https://doi.org/10.1176/appi.ajp.162.12.2263

Mento, C., Silvestri, M. C., Muscatello, M. R. A., Rizzo, A., Celebre, L., Praticò, M., \& Bruno, A. (2021). Psychological Impact of Pro-Anorexia and Pro-Eating Disorder Websites on Adolescent Females: A Systematic Review. International Journal of Environmental Research and Public Health, 18(4), 2186. doi: https://doi.org/10.3390/ ijerph18042186

Moreno, L. R., Domingo, J. P., Burguet, L. C., Leal, F. V., Marsá, M. D., RojoBofill, L., \& Aldana, L. L. (2012). Eating disorders: Considerations on nosology, etiology and treatment in the XXI century. Revista de Psiquiatría y Salud Mental (English Edition), 5(3), 197-204. doi: https://doi.org/10.1016/j.rpsmen.2012.02.002

Patching, J. \& Lawler, J. (2009). Understanding women's experiences of developing an eating disorder and recovering: a life-history approach. Nursing inquiry, 16(1), 10-21. doi: https://doi.org/10.1111/j.1440-1800.2009.00436.x

Sattar, N., McConnachie, A., Shaper, A. G., Blauw, G. J., Buckley, B. M., de Craen, A. J., \& Wannamethee, S. G. (2008). Can metabolic syndrome usefully predict cardiovascular disease and diabetes? Outcome data from two prospective studies. The Lancet, 371(9628), 1927-1935. doi: https://doi.org/10.1016/S0140-6736(08)60602-9

Syed-Abdul, S., Fernandez-Luque, L., Jian, W. S., Li, Y. C., Crain, S., Hsu, M. H., \& Liou, D. M. (2013). Misleading health-related information promoted through video-based social media: anorexia on YouTube. Journal of medical Internet research, 15(2), 30. doi: https://doi.org/10.2196/jmir.2237

Tiggemann, M., \& Slater, A. (2017). Facebook and body image concern in adolescent girls: A prospective study. International Journal of Eating Disorders, 50(1), 80-83. doi: https://doi.org/10.1002/eat.22640

Torres, R., Gerhart, N., \& Negahban, A. (2018). Epistemology in the era of fake news: An exploration of information verification behaviors among social networking site users. ACM SIGMIS Database: the DATABASE for Advances in Information Systems, 49(3), 78-97. doi: https://doi.org/10.1145/3242734.3242740

Wang, Y., McKee, M., Torbica, A., \& Stuckler, D. (2019). Systematic literature review on the spread of health-related misinformation on social media. Social Science E Medicine, 240, 112552. doi: https://doi.org/10.1016/j.socscimed.2019.112552

Zipfel, S., Giel, K., Bulik, C., Hay, P. \& Schmidt, U. (2015). Anorexia nervosa: aetiology, assessment, and treatment. The Lancet Psychiatry, 2, 1099-1111. doi: https:// doi.org/10.1016/S2215-0366(15)00356-9 